\title{
Continuous versus intermittent short-acting $\beta 2$-agonists nebulization as first-line therapy in hospitalized children with severe asthma exacerbation: a propensity score matching analysis
}

Prapasri Kulalert ${ }^{1}$, Phichayut Phinyo ${ }^{2,3^{*}}$ (D), Jayanton Patumanond ${ }^{3}$, Chutima Smathakanee ${ }^{4}$, Wantida Chuenjit ${ }^{4}$ and Sira Nanthapisal ${ }^{5}$

\begin{abstract}
Background: Short-acting $\beta 2$-agonist (SABA) nebulization is commonly prescribed for children hospitalized with severe asthma exacerbation. Either intermittent or continuous delivery has been considered safe and efficient. The comparative efficacy of these two modalities is inconclusive. We aimed to compare these two modalities as the first-line treatments.

Methods: An efficacy research with a retrospective cohort study design was conducted. Hospital records of children with severe asthma exacerbation admitted to Hat Yai Hospital between 2015 and 2017 were retrospectively collected. Children initially treated with continuous salbutamol $10 \mathrm{mg}$ per hour or intermittent salbutamol $2.5 \mathrm{mg}$ per dose over $1-4 \mathrm{~h}$ nebulization were matched one-to-one using the propensity score. Competing risk and risk difference regression was applied to evaluate the proportion of children who succeeded and failed the initial treatment. Restricted mean survival time regression was used to compare the length of stay (LOS) between the two groups.
\end{abstract}

Results: One-hundred and eighty-nine children were included. Of these children, 112 were matched for analysis (56 with continuous and 56 with intermittent nebulization). Children with continuous nebulization experienced a higher proportion of success in nebulization treatment (adjusted difference: $39.5,95 \% \mathrm{Cl} 22.7,56.3, p<0.001$ ), with a faster rate of success (adjusted SHR: $2.70,95 \% \mathrm{Cl} 1.73,4.22, p<0.001$ ). There was a tendency that LOS was also shorter (adjusted mean difference $-9.9 \mathrm{~h}, 95 \% \mathrm{Cl}-24.2,4.4, p=0.176$ ).

Conclusion: Continuous SABA nebulization was more efficient than intermittent nebulization in the treatment of children with severe asthma exacerbation.

Keywords: Status Asthmaticus, Pediatrics, Hospitalization, Nebulization, Bronchodilators, Propensity scores

\footnotetext{
* Correspondence: phichayutphinyo@gmail.com

${ }^{2}$ Department of Family Medicine, Faculty of Medicine, Chiang Mai University,

Chiang Mai, Thailand

${ }^{3}$ Center for Clinical Epidemiology and Clinical Statistics, Faculty of Medicine,

Chiang Mai University, Chiang Mai, Thailand

Full list of author information is available at the end of the article
}

(c) The Author(s). 2020 Open Access This article is licensed under a Creative Commons Attribution 4.0 International License, which permits use, sharing, adaptation, distribution and reproduction in any medium or format, as long as you give appropriate credit to the original author(s) and the source, provide a link to the Creative Commons licence, and indicate if changes were made. The images or other third party material in this article are included in the article's Creative Commons licence, unless indicated otherwise in a credit line to the material. If material is not included in the article's Creative Commons licence and your intended use is not permitted by statutory regulation or exceeds the permitted use, you will need to obtain permission directly from the copyright holder. To view a copy of this licence, visit http://creativecommons.org/licenses/by/4.0/ The Creative Commons Public Domain Dedication waiver (http://creativecommons.org/publicdomain/zero/1.0/) applies to the data made available in this article, unless otherwise stated in a credit line to the data. 


\section{Introduction}

Asthma exacerbation is one of the most common causes of hospitalization among children. It was observed that severe asthma exacerbation is increasing in children with asthma [1-3]. Intermittent nebulization with shortacting $\beta_{2}$-agonist (SABA), salbutamol $0.15-0.3 \mathrm{mg}$ per $\mathrm{kg}$, every one to 4 hours is the current first-line recommendation for hospitalized children with asthma exacerbation [4, 5]. However, children with severe asthma exacerbation may have suboptimal responses to first-line treatment and eventually require an escalation to more aggressive therapy (eg, continuous nebulization, intravenous salbutamol, or intravenous magnesium sulfate). Admission to the pediatric intensive care unit (PICU) is crucial for delivering and monitoring for side effects of these therapies $[2,3,6]$. When progression to lifethreatening respiratory failure occurs, endotracheal intubation and mechanical ventilation are needed. The results are asthma complications, prolonged hospital stays, and increased expenditures $[3,7,8]$. Prolonged PICU admission is also a very stressful experience and can be associated with post-traumatic stress disorder for both children and their parents $[9,10]$.

Continuous nebulization with SABA, salbutamol 10$25 \mathrm{mg}$ per hour or $0.5-1 \mathrm{mg}$ per $\mathrm{kg}$ per hour, has been recommended for children with asthma exacerbation who did not show adequate response to the first-line intermittent nebulization [11-13]. It was reported that some children with severe asthma exacerbation usually ended up with continuous nebulization therapy [2]. In the past, the continuous mode was limited to only lifethreatening cases and must be administered in the PICU due to the general perception of its potentially serious side effects when used in children (eg, diastolic hypotension, or arrhythmia). However, one study in 2014 reported various aspects of clinical improvements in severe asthma symptoms, lesser incidences of intensive care unit transfer, fewer patients requiring respiratory support, and sufficient safety when albuterol was administered continuously as the first-line treatments among children in non-PICU settings [14].

A proper asthma treatment strategy should aim to rapidly relieve the symptoms and reduce the chance of asthma complications. Early aggressive therapy may be an alternative solution. Since 2015, our hospital has developed a treatment protocol that allows the initiation of either continuous or intermittent nebulization as the first-line therapy in children with severe asthma exacerbation, depending on physicians' preferences. To the best of our knowledge, no study has yet compared the clinical efficacy between continuous and intermittent SABA nebulization as the first-line treatment in children with severe asthma exacerbation. We hypothesized that early administration of continuous SABA nebulization might increase the rate of successful treatment and could shorten the time to the resolution of asthmatic symptoms. The objective of our study was to investigate the clinical efficacy of continuous SABA nebulization as the first-line treatment instead of intermittent SABA nebulization in children with severe asthma exacerbation.

\section{Methods}

\section{Study design and patient cohort}

This therapeutic study was conducted based on a retrospective cohort of children with asthma exacerbation who were admitted at Hat Yai Hospital, a tertiary care hospital in Songkhla (province) between January 1, 2015 and December 31, 2017. Asthma diagnosis was identified by the International Classification of Diseases, Tenth Revision, and Clinical Modification codes (ICD-10) with the asthma discharge diagnosis codes J45 to 46. The requirement for informed consent was waived as the data were retrospectively collected and were anonymous. The study was approved by the Institutional Review Board of the Hat Yai Hospital (protocol number 33/2561) and the Faculty of Medicine, Thammasat University (MTU-ECES-0-061/61).

\section{Study participants}

All admission records during the study period were screened for eligibility. Only children who fulfilled all of the following criteria were included for analysis: 1) age between 1 and 15 years, 2) prior diagnosis of asthma or had at least two episodes of bronchodilator-responsive wheezing if no asthma diagnosis was documented, 3) having features of severe asthma exacerbation at initial admission, according to British Guidelines on the Management of Asthma (Table 1) [15], and 4) had been administered with continuous salbutamol nebulization 10 $\mathrm{mg} / \mathrm{h}$ or intermittent salbutamol nebulization $2.5 \mathrm{mg}$ per dose as first-line therapy. For accurate asthma diagnosis, children aged less than 12 months were excluded. Children were excluded if they were prescribed with other first-line therapy, such as adrenaline or $3 \% \mathrm{NaCl}$ nebulization, had any comorbidity eg, chronic cardiopulmonary diseases. We also excluded those who were referred from other hospitals, those who were referred to another hospital before complete resolution of asthma exacerbation, and those whose data on prognostic factors, emergency room (ER) treatment, clinical asthma severity on initial admission, and co-medications during admission were missing.

\section{Treatment groups}

Included children were coded as two groups according to their initial treatment at the pediatric ward. The salbutamol solution was prepared by diluting $2 \mathrm{~mL}$ of 
Table 1 Diagnosis of severe asthma exacerbation according to the British Guideline on the Management of Asthma for children in hospital

\begin{tabular}{ll}
\hline Age (years) & Severe asthma \\
\hline Age 1-5 & $\mathrm{SpO}_{2}<92 \%$ \\
& Too breathless to talk or eat \\
& $\mathrm{HR}>140 / \mathrm{min}$ \\
& $\mathrm{RR}>40 /$ min \\
& Use of accessory neck muscles \\
& Without features of life-threatening asthma \\
& $\mathrm{SpO} 2<92 \%$ \\
& $\mathrm{PEF} 33-50 \%$ best or predicted \\
Age $>5$ & Too breathless to talk or eat \\
& $\mathrm{HR}>125 /$ min \\
& $\mathrm{RR}>30 /$ min \\
& Use of accessory neck muscles \\
& Without features of life-threatening asthma \\
\hline
\end{tabular}

Abbreviations: HR Heart rate; PEF Peak expiratory flow; RR Respiratory rate; $\mathrm{SpO}_{2}$ Room air oxygen saturation

Ventolin Respirator solution ${ }^{\circ}(5 \mathrm{mg} / \mathrm{mL})$ in $28 \mathrm{~mL}$ of normal saline solution for both groups. For the first group, continuous nebulization of salbutamol was delivered by High Output Extended Aerosol Respiratory Therapy (HEART) via a face mask with an oxygen flow rate of $10 \mathrm{l}$ per minute at a concentration of $10 \mathrm{mg}$ per hour [16]. The second group was treated with intermittent nebulization of salbutamol. The dose of salbutamol was $1.5 \mathrm{mg}$ for children weighed $<10 \mathrm{~kg}$ and $2.5 \mathrm{mg}$ for children weighed $\geq 10 \mathrm{~kg}$. Salbutamol was delivered interval via a face mask with an oxygen flow rate of 6-81 per minute at 1 to $4 \mathrm{~h}$ intervals $[4,5]$.

Other than the first-line treatments, all children were provided with as standard asthma treatment: systemic corticosteroids given at recommended doses with supplemental oxygen to maintain oxygen saturation $\left(\mathrm{SpO}_{2}\right)$ $>95 \%[4,5,15]$. Additional therapy during admission was given as needed (eg, ipratropium bromide nebulization or subcutaneous terbutaline injection) depending on physician's judgment.

Like most hospitals, we employ a stepwise approach for the treatment of children with severe asthma exacerbation. The usual steps in escalating therapy in children with asthma exacerbation are as follows: 1) SABA delivered by intermittent nebulization, 2) continuous nebulization of SABA, 3) continuous intravenous terbutaline as adjunctive treatment, 4) If the children develop respiratory failure, endotracheal intubation and mechanical ventilator are used. Our step-down protocol is to provide each child with $10 \mathrm{mg}$ per hour of continuously nebulized salbutamol until the children's respiratory status improves. After that, the treatment would be changed to intermittent nebulization of salbutamol, 2.5 $\mathrm{mg}$ per dose, with sequentially decreasing of frequency (eg, every $1 \mathrm{~h}, 2 \mathrm{~h}, 3 \mathrm{~h}$, and $4 \mathrm{~h}$ ).

\section{Study endpoints}

The primary endpoint was the proportion of children who succeeded in the initial first-line treatment. Failure of initial treatment was defined as the escalation of treatment to more aggressive treatment. For intermittent nebulization therapy, treatment failure were defined as the requirement of at least one of the following treatments: continuous nebulization, intravenous terbutaline, continuous positive airway pressure, bi-level positive airway pressure, or mechanical ventilation. Children in the continuous nebulization group were considered to have failed if they subsequently required at least one of the following: intravenous terbutaline, ventilator support with continuous positive airway pressure, bi-level positive airway pressure, or mechanical ventilation. The secondary endpoint was the length of hospital stay (LOS).

\section{Pre-specified confounders Confounding by indication}

The characteristics that may affect physicians' treatment selection were the age of the children and the clinical severity of asthma exacerbation (eg, $\mathrm{SpO}_{2}$, respiratory rate, and pulse rate) [13]. Therefore, we defined age, respiratory rate, pulse rate, and oxygen saturation as confounding by indication and used them to derive the propensity scores.

\section{Prognostic factors}

Our prognostic factors were those that predisposed children toward the risk of asthma-related death or nearfatal asthma, PICU admission, or mechanical ventilation. These include 1) history of endotracheal intubation and mechanical ventilation for asthma exacerbation $[17,18]$, 2) $\geq 1$ asthma exacerbation (either hospitalization or ER visit for asthma) in the past 12 months [17], 3) not currently using controller medication [11, 17], 4) exacerbation triggered by pneumonia $[14,19], 5)$ subcutaneous injections of terbutaline at ER [14], or 6) obesity [20]. As not all children had the data on height, obesity was defined as a percentile of weight for age exceeding 90 [21].

\section{Pretreatment confounders and confounders by co- medications}

Medications for the treatment of asthma exacerbation during an ER and hospital admission may affect the children's clinical outcomes. Nebulized salbutamol $<3$ doses at ER and time to the first dose of systemic corticosteroid were defined as pre-treatment confounders [17]. Apart from the first-line SABA treatment during admission, co-medications (eg, subcutaneous injection of 
terbutaline, or ipratropium bromide nebulization) might be prescribed to some children depending on physicians' judgment and could potentially affect our study endpoints $[5,11,12]$. Two medications were defined as confounders by co-medications. Therefore, in this study, both pre-treatment confounders and confounders by comedications were considered during statistical analysis.

\section{Data collection}

Patient characteristics, including age, gender, weight, history of asthma diagnosis, history of using asthma controller medications, number of ER visits in the past 12 months, number of hospitalizations in the past 12 months, and history of intubation for asthma exacerbation were collected. Details on all medications for the treatment of asthma exacerbation at ER and during hospital admission were also collected. We recorded the time of initiation nebulization treatment, the time of admission to the ward or PICU, as well as the time of treatment cessation, and the time that physicians ordered discharge. Adverse side effects during nebulization (eg, nausea, vomiting, tremors, or diastolic hypotension) were observed and documented.

\section{Statistical analysis}

Data were analyzed using Stata version 16 (StataCorp, Lakeway, Texas, USA). For all statistical analyses, a twosided $p$-value less than 0.05 was considered as significant. Descriptive statistics were calculated for all clinical characteristics and relevant variables; frequencies were calculated for categorical variables and presented as percentages. Mean and standard deviation or median and interquartile range were calculated for continuous variables, as appropriate. We used standardized difference (STD) to measure the magnitude of differences in clinical characteristics, prognostic factors, and potential confounders between treatment groups, where an absolute STD of less than $10 \%$ was considered as no significant difference between groups [22].

As this was non-randomized therapeutic research, selection bias and imbalanced of prognostic determinants were likely to occur. We, therefore, performed a propensity score matching between the two groups before the estimation of the treatment effects. These techniques are regarded as acceptable for observational (non-randomized) studies [22-26]. We first calculated the propensity score to estimate the probability for each child to be initially treated with continuous nebulization by logistic regression. Our propensity score model included age, respiratory rate, pulse rate, and oxygen saturation on initial admission. The derived propensity scores were split into different blocks with a similar probability of being treated with continuous nebulization. Within each block, we matched the children who were treated with continuous nebulization to the children who were treated with intermittent nebulization with a one-to-one ratio. We then assessed the balance of baseline characteristics, prognostic factors, and potential confounders between the two groups after matching with STD.

Risk difference regression was applied to compare both the proportion of succeeding the first-line treatment and the proportion of failing the first-line treatment between treatment groups. As a failure of the first-line treatment could preclude the endpoint of interest and alter the probability of succeeding the first-line treatment, treatment failure is thus a competing risk in this clinical context. We used competing risk regression to estimate the cumulative incidence function for treatment success and treatment failure for continuous nebulization over intermittent nebulization. The results of competing risk analysis were presented as subdistribution hazard ratio (SHR), according to Fine and Gray [27]. In addition, the restricted mean survival time difference (RMST) was used to compare the differences in the LOS between both groups. The RMST analysis was based on flexible parametric modeling of log cumulative hazards with 3 d.f. for baseline hazard distribution and 1 d.f. for timetreatment interaction, as suggested by Royston and Parmar [28]. To entirely eliminate residual confounding, we adjusted all regression models with all remaining prognostic factors, pre-treatment confounders, and confounders by co-medications.

\section{Results}

A total of 568 medical charts of hospitalized children from January 2015 to December 2017 were screened. Of these, 276 did not fulfill the inclusion criteria: 154 had moderate asthma exacerbation, 41 were not diagnosed with asthma and had less than 2 episodes of bronchodilator-responsive wheezing, 34 were less than 12 months old, 22 had life-threatening asthma exacerbation, 21 were not diagnosed with asthma on admission (eg, bronchiolitis or anaphylaxis), and 4 were treated with different treatment protocols of SABA. Twohundred and ninety-two children with severe asthma exacerbation met the inclusion criteria. One-hundred and three children were excluded due to following reasons: 48 had missing data on emergency treatments, 21 had cardiopulmonary diseases, 15 had first-line therapy with other medications, 12 were referred to other hospitals before complete resolution of exacerbation, 4 had missing data on clinical asthma severity on initial admission, and 3 were referred from other hospitals (Fig. 1).

One hundred and eighty-nine children were included in this study, 60 were initially treated with continuous nebulization and 129 with intermittent nebulization. The comparison of patient clinical characteristics, prognostic factors, potential confounders, and propensity scores 


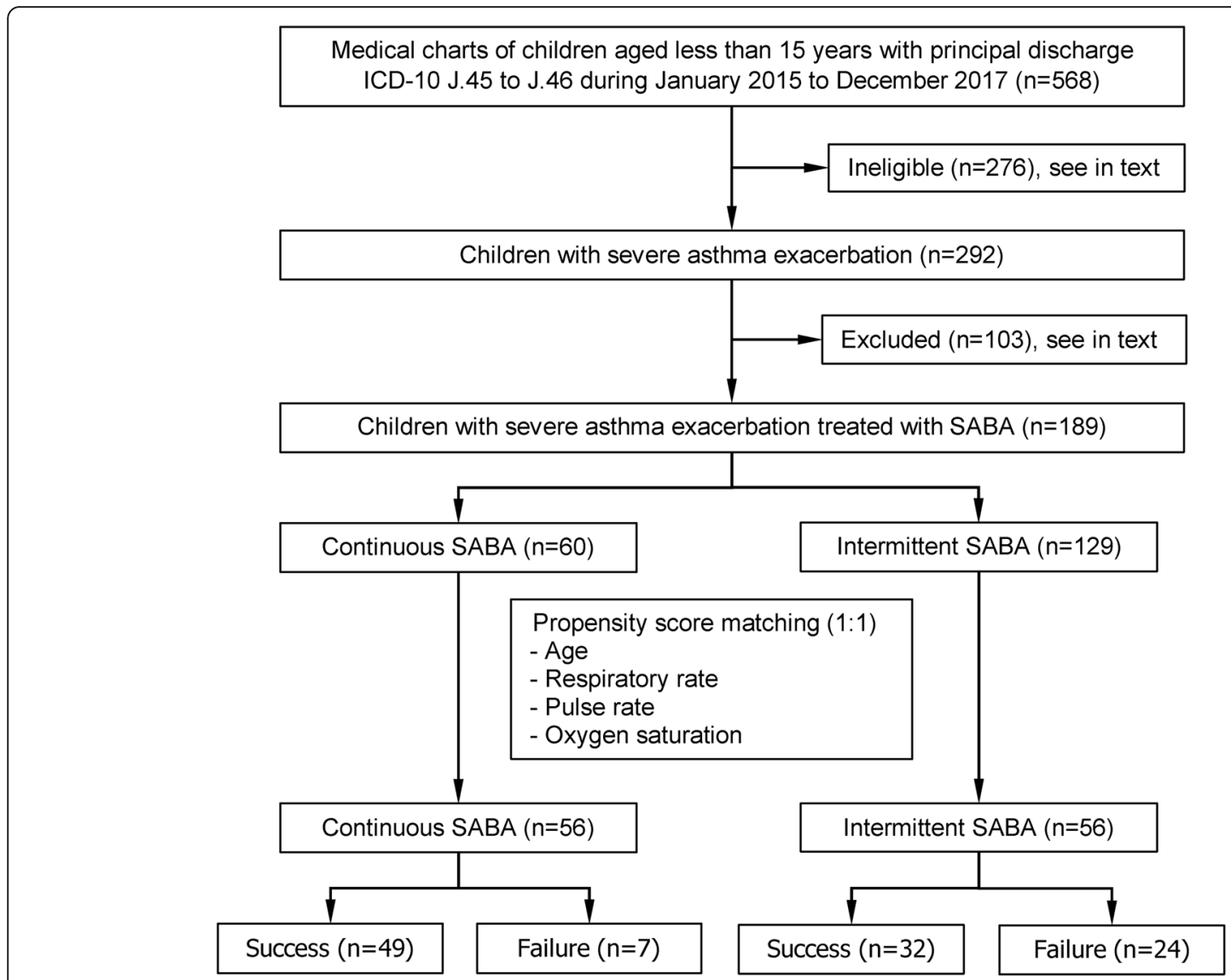

Fig. 1 Study flow diagram of the patient cohort

was shown in Table 2. Age, respiratory rate, pulse rate, and oxygen saturation on admission differed greatly between the two treatment groups with a standardized difference of more than $10 \%$ in all variables. After one-toone propensity score matching, we had 112 children divided evenly between initial continuous and intermittent nebulization (56:56). The propensity score model was shown in Table 3.

Table 2 also showed the clinical characteristics, prognostic factors, and potential confounders of the propensity score matched cohort. After matching, age, respiratory rate, pulse rate, and $\mathrm{SpO}_{2}$ were acceptably similar with a standardized difference less than $10 \%$. For prognostic factors, there were no notable discrepancies in the proportions of obese children, children with $\geq 1$ asthma exacerbation episode in the past 12 months, and children whose exacerbation were triggered by pneumonia. However, the proportion of children having a history of intubation for asthma and not currently using controller medication were significantly different (STD - 17.91 and STD 21.58, respectively).

Children in the continuous nebulization group had a lower proportion of nebulized salbutamol $<3$ doses at ER $(14.3 \%$ vs. $21.4 \%$, STD $=18.56 \%)$, a higher proportion of subcutaneous terbutaline injection $(16.1 \%$ vs. $7.1 \%$, STD $=-27.90 \%$ ), and lower time to the first dose of systemic corticosteroids (median time 53 vs. $70 \mathrm{~min}, \mathrm{STD}=$ $-11.40 \%)$. During admission, there was a significantly higher proportion of ipratropium bromide nebulization ( $41.1 \%$ vs. $30.4 \%$, STD $-22.3 \%)$ and subcutaneous injection of terbutaline $(50.0 \%$ vs. $12.5 \%$, STD $-87.67 \%)$ in the continuous nebulization group compared to the intermittent nebulization group.

Forty-nine children in the continuous nebulization group succeeded in the initial treatment, and only seven required escalating therapy with intravenous terbutaline injection. In the intermittent nebulization group, 32 children succeeded the initial treatment, 21 children were escalated to continuous nebulization with SABA, two were administered with intravenous terbutaline, and one child was mechanically ventilated. The results of all prespecified clinical endpoints were presented in Table 4. Children in the continuous nebulization group had a higher proportion of success compared to children in the intermittent nebulization group (adjusted risk difference $39.5,95 \%$ CI 22.7 to $56.3, p<0.001$ ). The rate of successful first-line nebulization treatment was also higher in the continuous nebulization group (adjusted 
Table 2 Clinical characteristics, prognostic factors, and potential confounders of the study patients

\begin{tabular}{|c|c|c|c|c|c|c|c|c|c|c|}
\hline \multirow[t]{3}{*}{ Characteristics } & \multicolumn{5}{|c|}{ Original cohort } & \multicolumn{5}{|c|}{ Propensity-matched cohort } \\
\hline & \multicolumn{2}{|c|}{$\begin{array}{l}\text { Continuous } \\
\text { nebulization } \\
(n=60)\end{array}$} & \multicolumn{2}{|c|}{$\begin{array}{l}\text { Intermittent } \\
\text { nebulization } \\
(n=129)\end{array}$} & \multirow{2}{*}{$\begin{array}{l}\text { STD } \\
(\%)\end{array}$} & \multicolumn{2}{|c|}{$\begin{array}{l}\text { Continuous } \\
\text { nebulization } \\
(n=56)\end{array}$} & \multicolumn{2}{|c|}{$\begin{array}{l}\text { Intermittent } \\
\text { nebulization } \\
(n=56)\end{array}$} & \multirow{2}{*}{$\begin{array}{l}\text { STD } \\
(\%)\end{array}$} \\
\hline & $n$ & (\%) & $n$ & $(\%)$ & & $n$ & (\%) & $n$ & (\%) & \\
\hline \multicolumn{11}{|l|}{ Clinical characteristics } \\
\hline Age (years), median (IQR) & 5.6 & $(3.3,8.9)$ & 4.2 & $(2.6,7.6)$ & -34.1 & 5.6 & $(3.3,8.7)$ & 5.6 & $(3.2,8.6)$ & -0.45 \\
\hline Male gender & 37 & $(61.7)$ & 84 & $(65.1)$ & 7.12 & 33 & $(58.9)$ & 33 & $(58.9)$ & 0 \\
\hline Weight for age (\%), median (IQR) & 23.6 & $\begin{array}{l}(6.7, \\
73.1)\end{array}$ & 28.4 & $\begin{array}{l}(3.9, \\
75.5)\end{array}$ & 2.87 & 27 & $\begin{array}{l}(10.5 \\
74.7)\end{array}$ & 23 & $\begin{array}{l}(2.9, \\
80.7)\end{array}$ & -2.87 \\
\hline \multicolumn{11}{|l|}{ Prognostic factors } \\
\hline Obesity & 10 & $(16.7)$ & 16 & $(12.4)$ & -12.04 & 10 & $(17.9)$ & 9 & $(16.1)$ & -4.72 \\
\hline Not currently using controller medication & 22 & $(36.7)$ & 59 & $(45.7)$ & 18.39 & 21 & $(37.5)$ & 27 & $(48.2)$ & 21.58 \\
\hline$\geq 1$ exacerbation in the past 12 months & 45 & $(75.0)$ & 97 & $(75.2)$ & 0.45 & 42 & $(75.0)$ & 41 & $(73.2)$ & -4.04 \\
\hline History of endotracheal intubation & 7 & $(11.7)$ & 12 & $(9.3)$ & -7.67 & 7 & $(12.5)$ & 4 & $(7.1)$ & -17.91 \\
\hline Exacerbation triggered by pneumonia & 18 & $(30.0)$ & 50 & (38.8) & 18.41 & 17 & $(30.4)$ & 19 & $(33.9)$ & 7.58 \\
\hline \multicolumn{11}{|l|}{ Treatment at emergency room } \\
\hline Nebulized salbutamol $<3$ doses & 8 & $(13.3)$ & 25 & $(19.4)$ & 16.31 & 8 & $(14.3)$ & 12 & $(21.4)$ & 18.56 \\
\hline Terbutaline subcutaneous injection & 10 & $(16.7)$ & 8 & $(6.2)$ & -33.11 & 9 & $(16.1)$ & 4 & $(7.1)$ & -27.90 \\
\hline $\begin{array}{l}\text { Time to first dose of systemic corticosteroids (min), median } \\
\text { (IQR) }\end{array}$ & 53 & $\begin{array}{l}(22, \\
155)\end{array}$ & 80 & $\begin{array}{l}(29, \\
175)\end{array}$ & 5.09 & 53 & $(23.5151)$ & 70 & $\begin{array}{l}(25, \\
149.5)\end{array}$ & -11.40 \\
\hline \multicolumn{11}{|l|}{ Clinical data on initial admission } \\
\hline Respiratory rate (per min), mean \pm SD & 40.8 & \pm 8.7 & 39.8 & \pm 7.5 & -12.50 & 40.7 & \pm 8.1 & 40.5 & \pm 8.0 & -1.78 \\
\hline Pulse rate (per min), mean \pm SD & 143.5 & \pm 16.9 & 147.2 & \pm 16.7 & 22.04 & 144.3 & \pm 16.5 & 143.6 & \pm 16.4 & -4.12 \\
\hline Oxygen saturation (\%), mean \pm SD & 93.9 & 3.2 & 93.5 & \pm 3.4 & -11.41 & 93.8 & \pm 3.3 & 93.6 & \pm 3.6 & -6.23 \\
\hline \multicolumn{11}{|l|}{ Co-medications during admission } \\
\hline Ipratropium bromide nebulization & 25 & $(41.7)$ & 37 & $(28.7)$ & -27.27 & 23 & $(41.1)$ & 17 & $(30.4)$ & -22.30 \\
\hline Terbutaline subcutaneous injection & 31 & $(51.7)$ & 15 & $(11.6)$ & -94.70 & 28 & $(50.0)$ & 7 & $(12.5)$ & -87.67 \\
\hline Propensity score, mean \pm SD & 0.37 & \pm 0.14 & 0.29 & \pm 0.12 & -62.56 & 0.35 & \pm 0.13 & 0.35 & \pm 0.13 & -0.62 \\
\hline
\end{tabular}

Abbreviation: IQR Interquartile range; SD Standard deviation; STD Standardized difference

Table 3 Derivation of propensity scores via multivariable logistic regression model

\begin{tabular}{|c|c|c|c|c|}
\hline Equation parameters & Coefficient & Standard error & 95\% confidence interval & $P$-value \\
\hline Age function $1^{\mathrm{a}}$ & -2.96 & 1.63 & $-6.15,0.23$ & 0.069 \\
\hline Age function $2^{\mathrm{a}}$ & 0.01 & 0.01 & $-0.01,0.01$ & 0.147 \\
\hline Respiratory rate function $1^{a}$ & $35,024.04$ & $17,178.24$ & $1355.31,68,692.77$ & 0.041 \\
\hline Respiratory rate function $2^{a}$ & $-11,823.00$ & 5542.34 & $-22,685.78,-960.22$ & 0.033 \\
\hline Pulse rate function $1^{\text {a }}$ & $-284,024.70$ & $707,345.10$ & $-1,670,396.00,1,102,346.00$ & 0.688 \\
\hline Pulse rate function $2^{a}$ & $66,655.37$ & $160,989.00$ & $-248,877.30,382,188.10$ & 0.679 \\
\hline Oxygen saturation function $1^{\mathrm{a}}$ & $-815,886.90$ & $2,937,831.00$ & $-6,573,930.00,4,942,156.00$ & 0.781 \\
\hline Oxygen saturation function $2^{a}$ & $197,629.00$ & $731,112.40$ & $-1,235,325.00,1,630,583.00$ & 0.787 \\
\hline Constant (intercept) & -6.93 & 43.00 & $-91.20,77.35$ & 0.872 \\
\hline
\end{tabular}


Table 4 Pre-specified study clinical endpoints

\begin{tabular}{|c|c|c|c|c|c|c|c|c|c|c|c|}
\hline \multirow[t]{3}{*}{ Clinical endpoints } & \multicolumn{2}{|c|}{$\begin{array}{l}\text { Continuous } \\
\text { nebulization } \\
(n=56)\end{array}$} & \multicolumn{2}{|c|}{$\begin{array}{l}\text { Intermittent } \\
\text { nebulization } \\
(n=56)\end{array}$} & \multicolumn{7}{|c|}{ Treatment effect (continuous vs. intermittent nebulization) } \\
\hline & \multirow[t]{2}{*}{$\mathrm{n}$} & \multirow[t]{2}{*}{$\%$} & \multirow[t]{2}{*}{$\mathrm{n}$} & \multirow[t]{2}{*}{$\%$} & \multirow[t]{2}{*}{ Clinical parameters } & \multicolumn{3}{|c|}{ Unadjusted analysis } & \multicolumn{3}{|c|}{ Adjusted analysis $^{a}$} \\
\hline & & & & & & Effect & $95 \% \mathrm{Cl}$ & $P$-value & Effect & $95 \% \mathrm{Cl}$ & $P$-value \\
\hline \multirow[t]{2}{*}{ Success } & 49 & 87.5 & 32 & 57.1 & Risk difference (\%) & 30.4 & $14.6,46.1$ & $<0.001$ & 39.5 & $22.7,56.3$ & $<0.001$ \\
\hline & & & & & SHR & 2.03 & $1.30,3.17$ & 0.002 & 2.70 & $1.72,4.22$ & $<0.001$ \\
\hline \multirow[t]{2}{*}{ Failure } & 7 & 12.5 & 24 & 42.9 & Risk difference (\%) & -30.4 & $-46.1,-14.6$ & $<0.001$ & -39.5 & $-56.3,-22.7$ & $<0.001$ \\
\hline & & & & & SHR & 0.25 & $0.11,0.58$ & 0.001 & 0.12 & $0.05,0.31$ & $<0.001$ \\
\hline LOS (hour), mean $\pm S D$ & 52.8 & \pm 28.2 & 59.1 & \pm 66.4 & RMST difference (mean) & -2.92 & $-14.54,8.70$ & 0.623 & -9.88 & $-24.18,4.42$ & 0.176 \\
\hline
\end{tabular}

Abbreviation: LOS Length of stay; RMST Restricted mean survival time; SHR Sub-distributional hazard ratio (under competing risk time-to-event

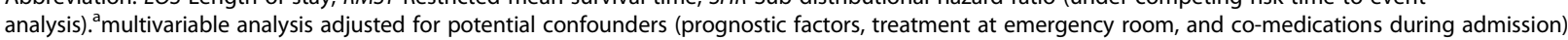

SHR: $2.70,95 \%$ CI 1.72 to $4.22, p<0.001$ ) (Fig. 2). The risk of failing initial treatment was also found to be lower in children treated with continuous nebulization (adjusted risk difference -39.5 , 95\% CI -56.3 to -22.7 , $p<0.001)$. The cumulative incidence of failing initial treatment was significantly lower in the continuous nebulization group (adjusted SHR: 0.12, 95\% CI 0.05 to 0.31 , $\mathrm{p}<0.001$ ) (Fig. 3).

The mean LOS was $52.8 \pm 28.2 \mathrm{~h}$ in the continuous nebulization group and $59.1 \pm 66.4 \mathrm{~h}$ in the intermittent nebulization group. After controlling for potential confounders, there was a tendency that the administration of continuous nebulization as a first-line treatment instead of intermittent nebulization could reduce the children's LOS by almost $10 \mathrm{~h}$ (adjusted RMST difference $-9.88 \mathrm{~h}, 95 \% \mathrm{CI}-$ 24.18 to $4.42, p=0.176$ ). For treatment-related adverse events, only one child who was treated with continuous nebulization reported nausea and vomiting. These symptoms disappeared after treatment cessation.

\section{Discussion}

According to our results, children with severe asthma exacerbation who were initially treated with continuous nebulization showed a significantly higher rate of treatment success and a lower chance of treatment failure and requirement of escalation to a more aggressive therapy compared to children who were treated with intermittent nebulization. Moreover, none of the children in the continuous nebulization group was intubated, whereas one of the children in the intermittent nebulization group was. Continuous nebulization as the first-line therapy also showed a tendency to reduce the LOS.

In terms of treatment efficacy, previous studies had reported and concluded the superiority of continuous nebulization with SABA over intermittent nebulization [29-32]. However, the patient's clinical characteristics, outcome measurements, and health care setting in these studies differed from ours. Two meta-analyses $[29,31]$ and one randomized trial [30] selectively included only children who

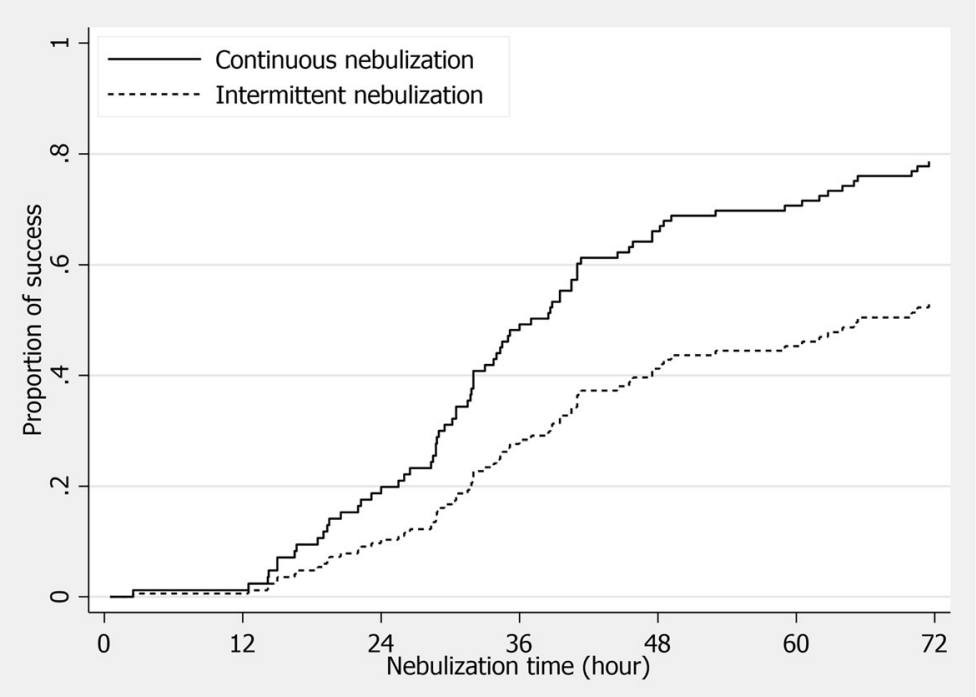

Fig. 2 Competing risk estimates for cumulative incidence of success in nebulization 


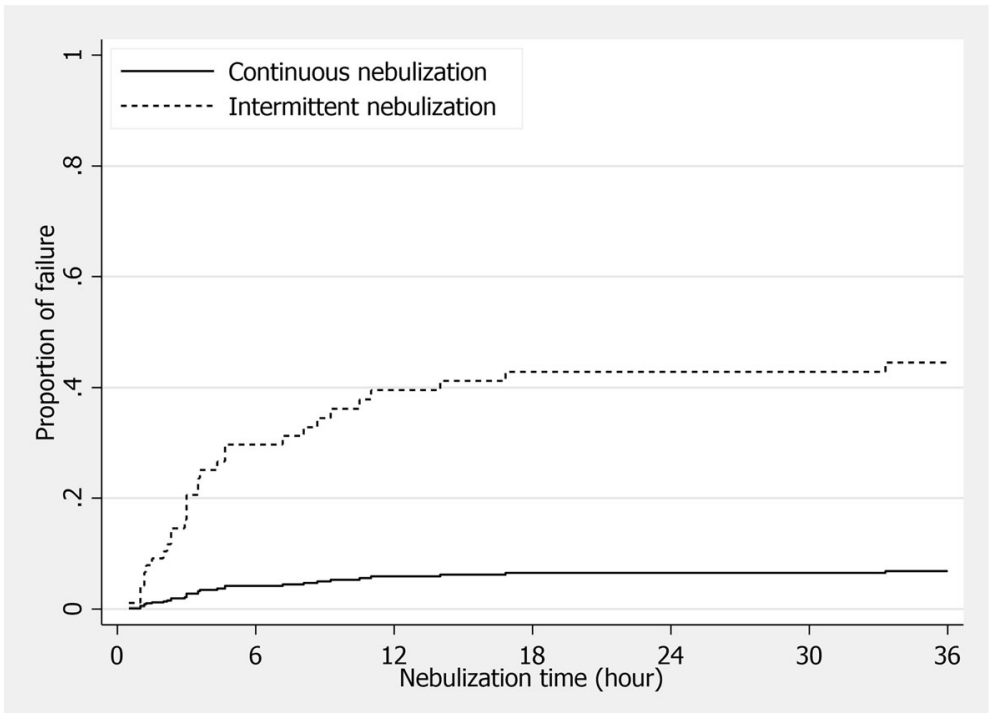

Fig. 3 Competing risk estimates for cumulative incidence of failure in nebulization

presented to the ER. It was concluded from these studies that children who were treated with continuous nebulization had an improvement in asthma score at $2 \mathrm{~h}$ after treatment and had shorter hospitalization. One study showed that continuous nebulization with albuterol significantly improved asthma outcomes in children with asthma exacerbation who presented with impending respiratory failure in a PICU setting [32]. The children in this study carried higher clinical severity of asthma exacerbation than the children within our study. Thus, the generalization of results from these studies to our clinical circumstance was not plausible. Another study evaluated the efficacy of continuous albuterol nebulization as the first-line treatment in children who were hospitalized with severe asthma exacerbation [14]. Only $5 \%$ of children with severe asthma exacerbation had clinical deterioration and required enhanced respiratory support (ie, using continuous positive airway pressure, or bi-level positive airway pressure) or transferred to PICU. No children were intubated. However, the study did not directly compare the efficacy of continuous nebulization among children with severe asthma exacerbation against that of intermittent nebulization.

Our study might be the first to directly compare the efficacy of continuous and intermittent SABA delivery as first-line treatment in children with severe asthma exacerbation admitted to general pediatric wards. Children with severe asthma exacerbation accounts for a large proportion of hospitalized children, and the trend seemed to be increasing [1-3]. These children usually have a suboptimal response to intermittent SABA nebulization and may require aggressive stepwise therapy, or may develop respiratory failure and end up with asthmarelated complications and prolonged hospital stay. Our study showed a lower proportion of children who required escalation of treatment and a higher rate of successful first-line treatment in the continuous nebulization group than in the intermittent nebulization group. In addition, no children in the continuous nebulization group developed respiratory failure and required endotracheal intubation. The reduction in the number of intubated cases would lead to fewer asthma complications, decreased health care costs, and shortened the $\operatorname{LOS}[3,7,8]$.

Children in the continuous nebulization group had a shorter LOS of approximately $10 \mathrm{~h}$, as compared to those in the intermittent nebulization group; however, the finding did not yield a statistical significance. This might be the consequence of limits in statistical power due to the small study size. However, considering the large volume of pediatric asthma hospitalizations, even a small reduction in the LOS can decrease a large economic burden, eg, a reduction of 0.5 days in median LOS could save $\$ 160$ million [33, 34]. However, as the burden of hospitalization due to asthma exacerbation varies by countries, and continuous therapy is more expensive than intermittent one. Future studies should aim to assess the cost-effectiveness of such approach with a larger population-based study.

Due to concerns regarding serious adverse effects of continuous nebulization with SABA (ie, diastolic hypotension, or arrhythmia), the treatment was strictly limited to children with a very severe or life-threatening asthma exacerbation who were also needed to be admitted to PICU before the administration of continuous therapy. These serious side effects are known to be dosedependent and commonly occur when given at a high 
dose $25 \mathrm{mg}$ per hour, $75 \mathrm{mg}$ per hour and $150 \mathrm{mg}$ per hour [35-37]. In this study, a lower dose, $10 \mathrm{mg}$ per hour, was used, which was relatively safe compared to the higher dose. There was only one child who developed nausea and vomiting. Previous studies also supported the clinical safety of the dosage used in this study [14, 37].

To estimate causal effect between applying continuous nebulization as the first-line therapy and the improvements in asthma endpoints from observational data, we used the propensity score method to minimize the presence of selection bias and balance the distribution of measured covariates that give rise to confounding by indication or confounding by contraindication [22]. In this study, propensity matching was used for the derivation of a comparable cohort of continuous and intermittent nebulization therapy $[22,38]$. The score was based on four pre-specified characteristics that influence the selection of treatments, either continuous or intermittent nebulization, by clinicians, which were age, respiratory rate, pulse rate, and oxygen saturation upon admission. After matching, the existing differences between groups became smaller; however, the remaining prognostic factors and potential confounders were imbalance with STD $>10 \%$. Concluding the treatment effect in the presence of a significant imbalance of covariates could compromise the result. Therefore, we performed double adjustment by multivariable adjustment of remaining prognostic factors and potential confounders with STD $>10 \%$ [39]. This approach could substantially eliminate residual confounding bias, increase statistical power [40], and improve the validity of causal estimate [41].

There were some limitations to be addressed. First, we could not perform peak expiratory flow rate (PEFR) measurements to assess the lung function and treatment response to all children who were older than 5 years old, as we did not have enough peak flow meter in the past. Besides, approximately half of our patients aged less than 5 years old. In this study, pulse oximetry, another objective measurement suggested by the Global Initiative for Asthma (GINA) guidelines [17], was used to assess the children before and after treatment. Second, the data used for analysis were retrospectively collected. All nebulization techniques were done according to our routine practice without standard operating procedures as in clinical trials. However, our health care team generally follows local guidelines on how to deliver and monitor the side effects of all medications. Thus, although the data might not be in a well-controlled environment, it did reflect the real-world effectiveness of both treatments in our setting. Third, the study size after propensity score matching was small and might not be adequate to power the multivariable regression analysis. However, as the treatment effect was substantial, the effect estimates were acceptably precise. Fourth, even after propensity score matching and multivariable adjustment of all measured confounders, residual confounding from unmeasured covariates may remain and could confound the treatment effects. A future randomized clinical trial to evaluate the efficacy of continuous nebulization therapy as the first-line treatment in hospitalized children with severe asthma exacerbation should be done to confirm the results of our study.

In conclusion, the administration of continuous salbutamol nebulization as first-line therapy in children with severe asthma exacerbation revealed a higher success rate and decreased in need for escalation of therapy. It should be considered as an alternative first-line treatment for hospitalized children with severe asthma exacerbation.

\section{Abbreviations \\ SABA: Short-acting $\beta_{2}$-agonist; PICU: Pediatric intensive care unit; ICD: International classification of diseases; ER: Emergency room; HEART: High output extended aerosol respiratory therapy; $\mathrm{SpO}_{2}$ : Oxygen saturation; STD: Standardized difference; SHR: Subdistribution hazard ratio; RMST: Restricted mean survival time; LOS: Length of hospital stay; PEFR: Peak expiratory flow rate; GINA: Global initiative for asthma}

\section{Acknowledgements}

The authors wish to thank Ms. Debra Kim Liwiski, an international instructor at Clinical Research Center, Faculty of Medicine, Thammasat University, and Ms. Lori Conover, Lecturer at the Language Institute Thammasat University (LITU) for language editing.

\section{Authors' contributions}

Conceptualization, P.K. and S.N.; methodology, P.P. and J.P.; software, P.P.; validation, P.P. and J.P.; formal analysis, P.K., P.P. and J.P.; investigation, P.K., C.S. and W.C.; resources, P.P.; data curation, P.K., C.S. and W.C.;

writing-original draft preparation, P.K.; writing—-review and editing, P.P. and J.P.; visualization, P.K.; supervision, J.P. and P.P.; project administration, K.P. and S.N.; All authors have read and agreed to the published version of the manuscript. The author (s) read and approved the final manuscript.

Funding

The authors declare no external funding.

\section{Availability of data and materials}

The datasets used and/or analyzed during the current study are available from the corresponding author on reasonable request.

\section{Ethics approval and consent to participate}

The Institutional Review Board and the Ethics Committee of Hat Yai Hospital (protocol number 33/2561) and the Faculty of Medicine, Thammasat University (MTU-EC-ES-0-061/61) approved the study protocol. The requirement for informed consent was waived as the data were retrospectively collected and were anonymous.

\section{Consent for publication}

Not applicable.

\section{Competing interests}

The authors declare no competing interests.

\section{Author details}

'Department of Clinical Epidemiology, Faculty of Medicine, Thammasat University, Pathum Thani, Thailand. ${ }^{2}$ Department of Family Medicine, Faculty of Medicine, Chiang Mai University, Chiang Mai, Thailand. ${ }^{3}$ Center for Clinical Epidemiology and Clinical Statistics, Faculty of Medicine, Chiang Mai

University, Chiang Mai, Thailand. ${ }^{4}$ Department of Pediatrics, Hat Yai Hospital, Songkhla, Thailand. ${ }^{5}$ Department of Pediatrics, Faculty of Medicine,

Thammasat University, Pathum Thani, Thailand. 
Received: 5 May 2020 Accepted: 21 June 2020

Published online: 02 July 2020

\section{References}

1. Hartman ME, Linde-Zwirble WT, Angus DC, Watson RS. Trends in admissions for pediatric status asthmaticus in New Jersey over a 15-year period. Pediatrics. 2010/09/30. 2010;126:e904-11.

2. Al-Eyadhy AA, Temsah MH, Alhaboob AA, Aldubayan AK, Almousa NA, Alsharidah AM, et al. Asthma changes at a pediatric intensive care unit after 10 years: Observational study. Ann Thorac Med. 2015/12/15. 2015;10:243-8.

3. Boeschoten SA, Buysse CMP, Merkus P, van Wijngaarden JMC, Heisterkamp SGJ, de Jongste JC, et al. Children with severe acute asthma admitted to Dutch PICUs: A changing landscape. Pediatr Pulmonol. 2018/04/11. 2018;53: 857-865.

4. Pardue Jones B, Fleming GM, Otillio JK, Asokan I, Arnold DH. Pediatric acute asthma exacerbations: Evaluation and management from emergency department to intensive care unit. J Asthma. 2016/04/27. 2016;53:607-17.

5. Camargo CA Jr, Rachelefsky G, Schatz M. Managing asthma exacerbations in the emergency department: summary of the National asthma education and prevention program expert panel report 3 guidelines for the management of asthma exacerbations. Proc Am Thorac Soc. 2009/08/14 2009:6:357-366.

6. Rehder KJ. Adjunct therapies for refractory status asthmaticus in children. Respir Care. 2017/05/27. 2017;62:849-65.

7. Carroll $\mathrm{CL}$, Zucker AR. The increased cost of complications in children with status asthmaticus. Pediatr Pulmonol. 2007/08/30. 2007:42:914-9.

8. Chiang BL, Hsieh CT, Wang LC, Lee JH, Yu HH, Lin YT, et al. Clinical course and outcome of children with status asthmaticus treated in a pediatric intensive care unit: a 15-year review. J Microbiol Immunol Infect. 2010/04/ 28. 2009;42:488-93.

9. Rees G, Gledhill J, Garralda ME, Nadel S. Psychiatric outcome following paediatric intensive care unit (PICU) admission: a cohort study. Intensive Care Med. 2004/04/28. 2004:30:1607-14

10. Bronner MB, Knoester H, Bos AP, Last BF, Grootenhuis MA. Follow-up after paediatric intensive care treatment: parental posttraumatic stress. Acta Paediatr. 2008/02/08. 2008;97:181-6

11. Fergeson JE, Patel SS, Lockey RF. Acute asthma, prognosis, and treatment. J Allergy Clin Immunol. 2016/08/25. 2017;139:438-47.

12. Nievas IF, Anand KJ. Severe acute asthma exacerbation in children: stepwise approach for escalating therapy in a pediatric intensive care unit. J Pediatr Pharmacol Ther. 2013/06/27. 2013;18:88-104.

13. Carroll CL, Sala KA. Pediatric status asthmaticus. Crit Care Clin. 2013/03/30. 2013;29:153-66

14. Kenyon CC, Fieldston ES, Luan X, Keren R, Zorc JJ. Safety and effectiveness of continuous aerosolized albuterol in the non-intensive care setting. Pediatrics. 2014/10/01. 2014;134:e976-82.

15. British Thoracic Society, Scottish Intercollegiate Guidelines Network. British guideline on the management of asthma. Thorax. 2014/10/18. 2014;69 Suppl 1:1-192.

16. Raabe OG, Wong TM, Wong GB, Roxburgh JW, Piper SD, Lee Jl. Continuous nebulization therapy for asthma with aerosols of beta2 agonists. Ann Allergy Asthma Immunol. 1998/07/01. 1998:80:499-508.

17. Global Initiative for Asthma. Global Strategy for Asthma Management and Prevention. [Internet]. 2019. Available from: https://ginasthma.org/wpcontent/uploads/2019/06/GINA-2019-main-report-June-2019-wms.pdf.

18. Alvarez GG, Schulzer M, Jung D, Fitzgerald JM. A systematic review of risk factors associated with near-fatal and fatal asthma. Can Respir J. 2005/08/19. 2005; 12:265-70

19. Grunwell JR, Travers C, Fitzpatrick AM. Inflammatory and Comorbid Features of Children Admitted to a PICU for Status Asthmaticus. Pediatr Crit Care Med. 2018/08/15. 2018:19:e585-94.

20. Okubo Y, Nochioka K, Hataya H, Sakakibara H, Terakawa T, Testa M. Burden of obesity on pediatric in patients with acute asthma exacerbation in the United States. J Allergy Clin Immunol Pr. 2016/07/04. 2016;4:1227-31.

21. Gamliel A, Ziv-Baran T, Siegel RM, Fogelman Y, Dubnov-Raz G. Using weight-for-age percentiles to screen for overweight and obese children and adolescents. Prev Med. 2015/09/09. 2015;81:174-9.

22. Austin PC. An introduction to propensity score methods for reducing the effects of confounding in observational studies. Multivar Behav Res. 2011/ 08/06. 2011;46:399-424.
23. Williamson EJ, Forbes A. Introduction to propensity scores. Respirology. 2014/06/04. 2014;19:625-35.

24. Haukoos JS, Lewis RJ. The propensity score. Jama. 2015/10/27. 2015;314 $1637-8$

25. Staffa SJ, Zurakowski D. Five steps to successfully implement and evaluate propensity score matching in clinical research studies. Anesth Analg. 2018/ 01/13. 2018;127:1066-73.

26. Garrido MM, Kelley AS, Paris J, Roza K, Meier DE, Morrison RS, et al. Methods for constructing and assessing propensity scores. Health Serv Res. 2014/05/ 02. 2014;49:1701-20

27. Fine JP, Gray RJ. A proportional hazards model for the subdistribution of a competing risk. J Am Stat Assoc. [American Statistical Association, Taylor \& Francis, Ltd. 1999:94:496-509.

28. Royston P, Parmar MKB. The use of restricted mean survival time to estimate the treatment effect in randomized clinical trials when the proportional hazards assumption is in doubt. Stat Med. 2011;30:2409-21.

29. Camargo CA Jr, Spooner $\mathrm{CH}$, Rowe BH. Continuous versus intermittent betaagonists in the treatment of acute asthma. Cochrane Database Syst Rev. 2003; 2003/10/30:;Cd001115

30. Khine H, Fuchs SM, Saville AL. Continuous vs intermittent nebulized albuterol for emergency management of asthma. Acad Emerg Med. 1996/ 11/01. 1996:3:1019-24.

31. Pollock M, Sinha IP, Hartling L, Rowe BH, Schreiber S, Fernandes RM. Inhaled short-acting bronchodilators for managing emergency childhood asthma: an overview of reviews. Allergy. 2016/09/03. 2017;72:183-200.

32. Papo MC, Frank J, Thompson AE. A prospective, randomized study of continuous versus intermittent nebulized albuterol for severe status asthmaticus in children. Crit Care Med. 1993/10/01. 1993;21:1479-86.

33. Shanley LA, Lin H, Flores $G$. Factors associated with length of stay for pediatric asthma hospitalizations. J Asthma. 2014/11/07. 2015;52:471-7.

34. HCUPnet. National estimates on use of hospitals by children from the HCUP Kids' Inpatient Database (KID). Agency of Healthcare Quality and Research. 2020. https://hcupnet.ahrq.gov. Accessed 5 May 2020.

35. Phumeetham S, Bahk TJ, Abd-Allah S, Mathur M. Effect of high-dose continuous albuterol nebulization on clinical variables in children with status asthmaticus. Pediatr Crit Care Med. 2015/01/07. 2015;16:e41-6.

36. Wisecup S, Eades S, Hashmi SS, Samuels C, Mosquera RA. Diastolic hypotension in pediatric patients with asthma receiving continuous albuterol. J Asthma. 2015/03/05. 2015:52:693-8.

37. Lin AT, Moore-Clingenpeel M, Karsies TJ. Comparison of two continuous nebulized albuterol doses in critically ill children with status asthmaticus. J Asthma 2019/05/24. 2019:1-7.

38. Deb S, Austin PC, Tu JV, Ko DT, Mazer CD, Kiss A, et al. A review of propensity-score methods and their use in cardiovascular research. Can J Cardiol. 2015/09/01. 2016;32:259-65.

39. Austin PC. Double propensity-score adjustment: a solution to design bias or bias due to incomplete matching. Stat methods med res. SAGE Publ Ltd STM. 2017:26:201-22.

40. Steyerberg EW. Clinical prediction models: a practical approach to development, validation, and updating: Springer; 2019.

41. Nguyen T-L, Collins GS, Spence J, Daurès J-P, Devereaux PJ, Landais P, et al. Double-adjustment in propensity score matching analysis: choosing a threshold for considering residual imbalance. BMC Med Res Methodol. 2017; 17:78.

\section{Publisher's Note}

Springer Nature remains neutral with regard to jurisdictional claims in published maps and institutional affiliations. 